\title{
EFEITOS CLÍNICOS E COMPORTAMENTAIS PROMOVIDOS PELA INJEÇÃO INTRAVENOSA DE MORFINA EM EQUINOS PURO SANGUE ÁRABE
}

\author{
Erica Cristina Bueno do Prado Guirro, ${ }^{1}$ João Henrique Perotta ${ }^{2}$ E \\ Carlos Augusto Araújo Valadẽo 3 \\ 1. Professora adjunta, UFPR, Campus Palotina. E-mail: ericaguirro@ufpr.br \\ 2. Mestrando do Programa de Pós-Graduação em Cirurgia Veterinária \\ 3. Professor adjunto do Departamento de Clínica e Cirurgia Veterinária, FCAV, UNESP.
}

\section{RESUMO}

O uso sistêmico de morfina em equinos é bastante controverso e ainda exige a realização de mais estudos. Na literatura não existem relatos sobre o uso intravenoso de morfina em cavalos da raça Puro Sangue Árabe e, assim, doze animais foram selecionados e divididos entre os grupos $\mathrm{GC}(5 \mathrm{~mL} \mathrm{NaCl} 09 \%$, iv) ou $\mathrm{GM}(0,1 \mathrm{mg} /$ Kg morfina, iv). Realizou-se aferição basal em T-20 e T-10, sendo os fármacos injetados em $\mathrm{T} 0$ e novas mensurações feitas em T30min, T60min, T90min, T2h, T3h, T6h, T12h e T24h. Os parâmetros avaliados incluíram frequência cardíaca e respiratória, temperatura retal, altura de cabeça em relação ao solo, ptose palpebral e labial, motilidade intestinal e ataxia. Além disso, verificaram-se o tempo de latência para urinar e defecar e a porcentagem de animais que urinaram ou defecaram. Não houve diferença significativa nos parâmetros avaliados, exceto na motilidade intestinal, que apresentou redução de T30 a T2h nos animais tratados com morfina. Assim, conclui-se que a aplicação intravenosa de morfina $(0,1 \mathrm{mg} / \mathrm{Kg})$ foi segura e não gerou alterações clínicas ou comportamentais severas nos cavalos Puro Sangue Árabe.

PALAVRAS-CHAVES: Equinos, morfina, Puro Sangue Árabe.

\section{ABSTRACT}

\section{CLINICAL AND BEHAVIORAL EFFECTS PROMOTED BY INTRAVENOUS INJECTION OF MORPHINE IN ARABIAN HORSES}

Systemic administration of morphine in horses is controversial and requires more investigation. In literature, there is no study about intravenous use of morphine in Arabian Horses; because of that, 12 animals were selected and distributed into two groups, GC $(5 \mathrm{~mL}$ $\mathrm{NaCl} 09 \%$, iv $)$ and $\mathrm{GM}(0,1 \mathrm{mg} / \mathrm{Kg}$ morphine, iv). Basal evaluation was performed in T-20 and T-10, drugs were injected in T0 and other measures were carried out in T30 min, T60 min, T90min, T2h, T3h, T6h, T12h and T24h. Parameters evaluated included heart and respiratory rate, body temperature, height of the head in relation to the ground, eye and lip drop, intestinal motility and ataxia. Besides, latency to urinate and defecate, and the percentage of animals which urinated or defecated were observed. There were no differences in the parameters, except in intestinal motility that was reduced from $\mathrm{T} 30$ to T2h in GM. So, we concluded that intravenous application of morphine $(0.1 \mathrm{mg} / \mathrm{Kg})$ was safe and did not promot clinical or behavioral changes in Arabian Horses.

KEYWORDS: Arabian Horses, horses, morphine. 


\section{INTRODUÇÃO}

O bem-estar animal é um tema atual na medicina veterinária e, neste contexto, se insere a minimização da dor, sensação capaz de desequilibrar a homeostase e gerar alterações adaptativas autonômicas, endócrinas e comportamentais (DANNEMAN, 1997), além de dificultar a realização de procedimentos terapêuticos, especialmente com grandes animais. A dor é definida pela Associação Internacional para o Estudo da Dor como "uma experiência sensitiva e emocional desagradável associada à lesão tecidual real ou potencial".

Diversos estudos evidenciam que ainda há marcado interesse na busca por terapias analgésicas que possam minimizar a dor nos equinos. Além disso, um cavalo excitado em decorrência da dor pode lesionarse mais frequentemente que o animal sem dor, calmo, relaxado e com suporte físico adequado (TAYLOR \& CLARKE, 1999; TAYLOR et al., 2002; TAYLOR, 2005).

A avaliação da dor é apontada como uma das principais dificuldades no manuseio da dor pósoperatória, desde recém-nascidos (SILVA et al., 2007) até crianças menores de seis anos (MENEZES \& GOZZANI, 2002), e semelhante dificuldade é encontrada na medicina veterinária. HELLYER (2002) acredita que as falhas no reconhecimento da dor em equinos levam muitos clínicos a não prescrevem analgésicos, por não acreditarem que seu paciente esteja em estado álgico.

Os opioides são analgésicos amplamente empregados no combate aos processos dolorosos (SAMMARCO et al., 1996; PASCOE \& TAYLOR, 2003), visto que tais fármacos interagem com receptores opioides, principalmente dos tipos $\mu$ (mu), $\kappa$ (kappa) e $\delta$ (delta) (GAVERIAUX-RUFF \& KIEFFER, 1999; RANG et al., 2004) e desencadeiam diversos mecanismos moleculares que levam à analgesia (DICKENSON \& SULLIVAN, 1987; SCHULTZ \& GROSS, 2001).

A morfina é o fármaco padrão dos agonistas de receptores $\mu$ cuja dose terapêutica para administração sistêmica varia entre 0,04 a $0,1 \mathrm{mg} / \mathrm{kg}$, em equinos (MUIR III, 1991; TAYLOR \& CLARKE, 1999; MUIR III, 2001). Todavia, esse uso tem sido desencorajado nesta espécie, dada a possibilidade de ocorrerem efeitos colaterais severos (TAYLOR et al., 2002), já que existem relatos de aumento da frequência cardíaca, aumento das pressões arterial e venosa central, tremores, depressão respiratória, redução da temperatura corporal, sudorese, rigidez da marcha, efeito inibitório sobre a motilidade intestinal e aumento da atividade locomotora (TOBIN, 1978; KALPRAVIDH et al., 1984; MUIR III, 1991; THURMON et al., 1996; TAYLOR \& CLARKE, 1999; MUIR III et al., 2001; HUBBELL, 2006). Assim, TAYLOR (2005) afirma que ainda há necessidade de se investigar melhor o uso sistêmico de morfina na rotina clínica de equinos.

THURMON et al. (1996) citam que os opioides podem apresentar diferenças quanto à ação inclusive dentro de uma espécie. Além disso, algumas raças respondem de modo distinto à ação de determinado fármaco, geralmente conforme a sensibilidade ao agente empregado. Visto que na literatura pertinente não existem relatos a respeito do uso de morfina em cavalos da raça Puro Sangue Árabe (PSA), com este estudo objetivou-se avaliar os efeitos clínicos e comportamentais promovidos pela injeção intravenosa de morfina nesta raça.

\section{MATERIAL E MÉTODOS}

Este trabalho foi desenvolvido de acordo com as normas éticas, após ser aprovado pelo Comitê de Ética e Bem-Estar Animal da FCAV - UNESP (protocolo n. ${ }^{\circ}$ 009974-05).

Doze equinos da raça Puro Sangue Árabe, com idade entre quatro e seis anos, peso médio de $377 \pm$ $27 \mathrm{Kg}$, sendo seis machos castrados e seis fềmeas não prenhes e fora do período de estro foram submetidos a exame físico, classificados como hígidos e, portanto, selecionados. Distribuíram-se os animais aleatoriamente nos grupos $\mathrm{GC}(5 \mathrm{~mL}, \mathrm{NaCl} 0,9 \%$, iv) ou GM $(0,1 \mathrm{mg} / \mathrm{Kg}$ morfina, iv), sendo cada grupo composto por três machos e três fêmeas.

Previamente à administração de morfina, realizaram-se duas aferições basais (T-20min e T-10min) de frequência cardíaca ( $\mathrm{FC}$, em bpm), frequência respiratória $(f, \mathrm{em} \mathrm{mpm})$, temperatura retal $\left(\mathrm{TR}, \mathrm{em}{ }^{\circ} \mathrm{C}\right)$, altura de cabeça em relação ao solo (AC, em \%), ptose palpebral e/ou labial (escores), motilidade intestinal (escores) e ataxia (escores).

O parâmetro altura de cabeça em relação ao solo foi medido em porcentagem de manutenção da altura da cabeça em relação ao solo após comparação com 
o tempo basal, pois dessa forma foi possível evitar discrepâncias entre as diferenças de alturas dos animais. Aferiu-se a motilidade intestinal por meio de auscultação nos quadrantes superior direito (QSD), superior esquerdo (QSE), inferior direito (QID) e inferior esquerdo (QIE). O QSD refere-se ao ceco e, portanto, aferiu-se a descarga da válvula ileocecal por cinco minutos, sendo considerada como normomotilidade a ocorrência de duas a quatro descargas ileocecais completas neste intervalo de tempo. Nos demais quadrantes, realizaram-se apenas avaliação qualitativa durante um minuto. Os escores utilizados na avaliação da motilidade intestinal foram: 0 - ausência de motilidade; 1 - movimentos intestinais incompletos; 2-hipomotilidade; 3-normomotilidade; 4-hipermotilidade.

Mediram-se o parâmetro ptosepalpebral e o ptoselabial conforme a porcentagem de animais que apresentaram ptose. A ataxia foi medida em escores, segundo adaptação da escala utilizada por BRYANT et al. (1991) em equinos 0 (ausência) - estabilidade; 1 (leve) - redução de estabilidade com alguma movimentação lateral; 2 (moderada) - movimentação lateral mais intensa, tendência à inclinação; 3 (severa) - apoio no tronco, membros pélvicos cruzados, flexões súbitas e frequentes das articulações carpais.

Em T0 realizou-se a administração dos fármacos conforme o grupo experimental e novas mensurações foram feitas em T30min, T60min, T90min, T2h, T3h, T6h, T12h e T24h. Além disso, verificaram-se o tempo de latência para urinar e defecar e, também, a porcentagem de animais que urinaram ou defecaram após a administração dos fármacos, até três horas de observação.

Os dados foram submetidos à análise estatística conforme a característica das variáveis. Utilizou-se ANOVA de uma via com repetições múltiplas, seguido pelo teste de Dunnett entre tempos e pelo teste Student-Newman-Keuls entre grupos $(\mathrm{p} \leq 0,05)$, para avaliar $\mathrm{FC}, f, \mathrm{~T}^{\circ}$ e $\mathrm{AC}$; ANOVA de uma via $(\mathrm{p} \leq 0,05)$, na análise da latência para urinar e defecar; KruskalWallis ANOVA, em blocos ( $\mathrm{p} \leq 0,05)$, na comparação da porcentagem de animais que urinaram e defecaram; Friedman ANOVA, em blocos com repetições múltiplas, seguido pelo teste de Dunnett entre tempos e pelo teste Student-Newman-Keuls entre grupos $(p \leq 0,05)$ na análise dos dados relacionados à ptosepalpebral ou labial, motilidade intestinal e ataxia.

\section{RESULTADOS E DISCUSSÃO}

Não houve alteração significativa entre tempos e entre grupos nos valores de frequência cardíaca, frequência respiratória, temperatura retal, altura de cabeça em relação ao solo, ptosepalpebral, ptoselabial e ataxia, conforme está ilustrado nas Tabelas 1 e 2 .

TABELA 1. Valores de frequência cardíaca $(\mathrm{FC})$, frequência respiratória $(f)$, temperatura retal $\left(\mathrm{T}^{\circ}\right)$ e altura da cabeça em relação ao solo (AC) encontrados nos equinos da raça Puro Sangue Árabe tratados com $\mathrm{NaCl}$ 0,9\% ou morfina

\begin{tabular}{ccccccccc}
\hline \multirow{2}{*}{ Tempo } & \multicolumn{2}{c}{ FC (bpm) } & \multicolumn{2}{c}{$f(\mathrm{mpm})$} & \multicolumn{2}{c}{$\mathrm{T}^{\circ}\left({ }^{\circ} \mathrm{C}\right)$} & \multicolumn{2}{c}{$\mathrm{AC}(\%)$} \\
\cline { 2 - 8 } & GC & GM & GC & GM & GC & GM & GC & GM \\
\hline T-20 & $40,7 \pm 3,0$ & $38,0 \pm 2,7$ & $26,7 \pm 3,2$ & $23,3 \pm 5,1$ & $37,5 \pm 0,1$ & $37,1 \pm 0,2$ & $100 \pm 0$ & $100 \pm 0$ \\
T-10 & $39,0 \pm 3,0$ & $35,3 \pm 2,3$ & $24,3 \pm 4,4$ & $21,3 \pm 2,6$ & $37,7 \pm 0,1$ & $37,4 \pm 0,2$ & $100 \pm 0$ & $100 \pm 0$ \\
T0 & $41,0 \pm 3,0$ & $43,7 \pm 2,2$ & $26,7 \pm 3,0$ & $30,7 \pm 1,9$ & $37,8 \pm 0,1$ & $37,4 \pm 0,2$ & $100 \pm 0$ & $100 \pm 0$ \\
T30 & $42,0 \pm 2,5$ & $36,0 \pm 1,9$ & $27,0 \pm 2,7$ & $29,7 \pm 3,8$ & $37,7 \pm 0,1$ & $37,6 \pm 0,2$ & $100 \pm 0$ & $100 \pm 0$ \\
T60 & $41,0 \pm 3,3$ & $37,0 \pm 2,0$ & $28,7 \pm 2,3$ & $32,3 \pm 3,0$ & $37,8 \pm 0,1$ & $37,6 \pm 0,2$ & $100 \pm 0$ & $100 \pm 0$ \\
T90 & $37,7 \pm 2,2$ & $37,3 \pm 2,0$ & $25,3 \pm 3,2$ & $34,4 \pm 4,0$ & $37,8 \pm 0,1$ & $37,6 \pm 0,3$ & $100 \pm 0$ & $96,7 \pm 3,3$ \\
T2h & $37,3 \pm 2,4$ & $38,3 \pm 2,3$ & $26,8 \pm 3,0$ & $29,3 \pm 4,0$ & $37,7 \pm 0,1$ & $37,5 \pm 0,3$ & $100 \pm 0$ & $100 \pm 0$ \\
T3h & $44,3 \pm 4,1$ & $42,0 \pm 2,2$ & $32,7 \pm 4,6$ & $31,7 \pm 2,6$ & $37,9 \pm 0,1$ & $37,7 \pm 0,2$ & $100 \pm 0$ & $100 \pm 0$ \\
T6h & $47,3 \pm 5,1$ & $42,2 \pm 5,5$ & $27,0 \pm 1,8$ & $31,7 \pm 2,2$ & $37,8 \pm 0,1$ & $37,7 \pm 0,1$ & $100 \pm 0$ & $100 \pm 0$ \\
T12h & $45,3 \pm 5,0$ & $42,0 \pm 3,5$ & $23,3 \pm 2,4$ & $24,7 \pm 3,6$ & $37,8 \pm 0,1$ & $37,7 \pm 0,1$ & $100 \pm 0$ & $100 \pm 0$ \\
T24h & $46,0 \pm 4,2$ & $38,3 \pm 2,7$ & $28,4 \pm 5,3$ & $27,3 \pm 3,9$ & $38,1 \pm 0,1$ & $37,9 \pm 0,2$ & $100 \pm 0$ & $100 \pm 0$ \\
\hline
\end{tabular}

* Diferente do valor de T-20, segundo o teste ANOVA, de uma via com repetições múltiplas seguido pelo teste de Dunnett ( $\mathrm{p} \leq 0,05$ ). 
TABELA 2. Escores de ptosepalpebral, ptoselabial e ataxia apresentados pelos equinos da raça Puro Sangue Árabe tratados com $\mathrm{NaCl}$ $0,9 \%$ ou morfina

\begin{tabular}{ccccccc}
\hline \multirow{2}{*}{ Tempo } & \multicolumn{2}{c}{ Ptose palpebral } & \multicolumn{2}{c}{ Ptose labial } & \multicolumn{2}{c}{ Ataxia } \\
\cline { 2 - 6 } & GC & GM & GC & GM & GC & GM \\
\hline T-20 & $0 \pm 0$ & $0 \pm 0$ & $0 \pm 0$ & $0 \pm 0$ & $0 \pm 0$ & $0 \pm 0$ \\
T-10 & $0 \pm 0$ & $0 \pm 0$ & $0 \pm 0$ & $0 \pm 0$ & $0 \pm 0$ & $0 \pm 0$ \\
T0 & $0 \pm 0$ & $16,7 \pm 16,7$ & $0 \pm 0$ & $0 \pm 0$ & $0 \pm 0$ & $0 \pm 0$ \\
T30 & $0 \pm 0$ & $33,3 \pm 21,1$ & $0 \pm 0$ & $0 \pm 0$ & $0 \pm 0$ & $0 \pm 0$ \\
T60 & $0 \pm 0$ & $50,0 \pm 22,4$ & $0 \pm 0$ & $0 \pm 0$ & $0 \pm 0$ & $0 \pm 0$ \\
T90 & $0 \pm 0$ & $16,7 \pm 16,7$ & $0 \pm 0$ & $0 \pm 0$ & $0 \pm 0$ & $0 \pm 0$ \\
T2h & $0 \pm 0$ & $0,0 \pm 0,0$ & $0 \pm 0$ & $0 \pm 0$ & $0 \pm 0$ & $0 \pm 0$ \\
T3h & $0 \pm 0$ & $0 \pm 0$ & $0 \pm 0$ & $0 \pm 0$ & $0 \pm 0$ & $0 \pm 0$ \\
T6h & $0 \pm 0$ & $0 \pm 0$ & $0 \pm 0$ & $0 \pm 0$ & $0 \pm 0$ & $0 \pm 0$ \\
T12h & $0 \pm 0$ & $0 \pm 0$ & $0 \pm 0$ & $0 \pm 0$ & $0 \pm 0$ & $0 \pm 0$ \\
T24h & $0 \pm 0$ & $0 \pm 0$ & $0 \pm 0$ & $0 \pm 0$ & $0 \pm 0$ & $0 \pm 0$ \\
\hline
\end{tabular}

Quanto à motilidade intestinal, em GC não houve variação entre tempos, ao passo que GM apresentou redução significativa em relação a T-20 em T30, T60, T90 e T2h nos quadrantes superior direito, inferior direito e inferior esquerdo. Entre grupos, os valores de GM foram inferiores ao de GC nos tempos T60, T90 e T2h no quadrante superior e inferior esquerdo e nos tempos T30, T60, T60 e T2h no quadrante inferior direito. Esses achados estão ilustrados na Tabela 3.

TABELA 3. Escores de motilidade intestinal verificados no quadrante superior direito (QSD), superior esquerdo (QSE), inferior direito (QID) e inferior esquerdo (QIE) apresentados pelos equinos da raça Puro Sangue Árabe tratados com $\mathrm{NaCl}$ 0,9\% ou morfina

\begin{tabular}{|c|c|c|c|c|c|c|c|c|}
\hline \multirow{2}{*}{ Tempo } & \multicolumn{2}{|c|}{ QSD } & \multicolumn{2}{|c|}{ QSE } & \multicolumn{2}{|c|}{ QID } & \multicolumn{2}{|c|}{ QIE } \\
\hline & GC & GM & GC & GM & GC & GM & GC & GM \\
\hline $\mathrm{T}-20$ & $1,5 \pm 0,3$ & $1,8 \pm 0,4$ & $2,0 \pm 0,0$ & $1,7 \pm 0,3$ & $1,8 \pm 0,4$ & $2,0 \pm 0,3$ & $2,2 \pm 0,2$ & $1,8 \pm 0,2$ \\
\hline $\mathrm{T}-10$ & $1,3 \pm 0,3$ & $1,0 \pm 0,4$ & $2,2 \pm 0,2$ & $1,7 \pm 0,2$ & $1,5 \pm 0,3$ & $1,2 \pm 0,3$ & $2,0 \pm 0,3$ & $1,8 \pm 0,2$ \\
\hline T0 & $1,2 \pm 0,2$ & $0,8 \pm 0,3$ & $2,0 \pm 0,4$ & $0,8 \pm 0,3$ & $1,3 \pm 0,2$ & $1,2 \pm 0,2$ & $1,7 \pm 0,2$ & $1,0 \pm 0,4$ \\
\hline $\mathrm{T} 30$ & $1,2 \pm 0,2$ & $0,2 \pm 0,2^{*}$ & $1,7 \pm 0,3$ & $0,7 \pm 0,3$ & $1,7 \pm 0,3$ & $0,5 \pm 0,2 *$ & $1,5 \pm 0,2$ & $0,7 \pm 0,3^{*}$ \\
\hline T60 & $1,5 \pm 0,2$ & $0,5 \pm 0,2 *$ & $2,0 \pm 0,3$ & $0,3 \pm 0,2$ & $1,7 \pm 0,2$ & $0,5 \pm 0,2^{*}$ & $1,7 \pm 0,2$ & $0,3 \pm 0,2^{*}$ \\
\hline T90 & $1,2 \pm 0,2$ & $0,3 \pm 0,2 *$ & $1,7 \pm 0,3$ & $0,5 \pm 0,2$ & $1,7 \pm 0,2$ & $0,5 \pm 0,2 *$ & $1,8 \pm 0,3$ & $0,7 \pm 0,2^{*}$ \\
\hline $\mathrm{T} 2 \mathrm{~h}$ & $1,3 \pm 0,3$ & $0,3 \pm 0,2 *$ & $2,2 \pm 0,2$ & $0,5 \pm 0,3$ & $2,0 \pm 0,0$ & $0,7 \pm 0,3^{*}$ & $2,0 \pm 0,3$ & $0,8 \pm 0,4^{*}$ \\
\hline $\mathrm{T} 3 \mathrm{~h}$ & $1,5 \pm 0,2$ & $1,5 \pm 0,5$ & $2,0 \pm 0,3$ & $1,3 \pm 0,2$ & $1,8 \pm 0,2$ & $1,7 \pm 0,4$ & $1,7 \pm 0,2$ & $1,5 \pm 0,2$ \\
\hline T6h & $1,8 \pm 0,2$ & $2,0 \pm 0,3$ & $1,8 \pm 0,2$ & $1,2 \pm 0,3$ & $2,0 \pm 0,3$ & $1,8 \pm 0,4$ & $2,2 \pm 0,2$ & $1,5 \pm 0,2$ \\
\hline $\mathrm{T} 12 \mathrm{~h}$ & $2,2 \pm 0,3$ & $1,8 \pm 0,2$ & $2,0 \pm 0,4$ & $1,5 \pm 0,3$ & $2,2 \pm 0,2$ & $1,8 \pm 0,2$ & $2,2 \pm 0,5$ & $2,0 \pm 0,3$ \\
\hline $\mathrm{T} 24 \mathrm{~h}$ & $1,5 \pm 0,4$ & $1,8 \pm 0,4$ & $1,7 \pm 0,2$ & $1,5 \pm 0,3$ & $2,0 \pm 0,4$ & $2,0 \pm 0,3$ & $1,7 \pm 0,2$ & $1,7 \pm 0,3$ \\
\hline
\end{tabular}

* Diferente do valor de T-20, segundo o teste Friedman ANOVA, em blocos com repetições múltiplas, seguido pelo teste de Dunnett (p<0,05). A área em cinza apresenta valor de GM menor que GC, segundo o teste Friedman ANOVA, em blocos com repetições múltiplas, seguido pelo teste StudentNewman-Keuls $(\mathrm{p}<0,05)$. 
Durante as três primeiras horas após a injeção dos fármacos, nenhum animal urinou e, portanto, a latência para urinar foi superior a 180 minutos em ambos os grupos. Quanto à defecação, $\mathrm{GC}$ apresentou taxa de defecação de $66,6 \%$ e latência de $69,7 \pm 35 \mathrm{~min}$, ao passo que GM apresentou taxa de defecção em $33,3 \%$ e latência de $143,3 \pm 23,3 \mathrm{~min}$. Nota-se que a administração de morfina reduziu a porcentagem de animais que defecaram e houve aumento do período de latência, todavia sem diferença estatística.

A morfina reduziu a motilidade de modo significativo no intestino grosso e, mesmo que sem diferença estatística, no intestino delgado. Essas observações decorrem do efeito promovido pelo agonistas mu, como é o caso da morfina, que diminui a motilidade do intestino grosso por até 120 minutos (THURMON et al., 1996), exatamente como foi verificado. TAYLOR (2005) salienta a necessidade de se investigar melhor os efeitos do uso sistêmico de morfina, em equinos, e o presente estudo mostrou que a injeção intravenosa de morfina $(0,1 \mathrm{mg} / \mathrm{kg}) \mathrm{em}$ cavalos PSA foi segura e causou apenas alterações leves e transitórias na motilidade intestinal, que foi restaurada após a segunda hora de avaliação, sem que os cavalos apresentassem outras manifestações. A inexistência de efeitos colaterais maiores em equinos tratados com $0,1 \mathrm{mg} / \mathrm{kg}$ morfina corrobora os resultados apontados por CAMARGO et al. (2005), que também não observaram alterações da motilidade intestinal. Alguns dos autores que citam a instalação de efeitos colaterais após o uso intravenoso de morfina referem-se ao emprego de doses superiores a $0,3 \mathrm{mg} / \mathrm{kg}$ (KALPRAVIDH, 1984; THURMON et al., 1996; HUBBELL, 2006). Obviamente, ainda é preciso avaliar melhor o uso da morfina em cavalos portadores de distúrbios gastrintestinais, em especial nos equinos que apresentem síndrome cólica.

Embora a porcentagem de animais que defecaram e a latência para defecar não tenham apresentado redução significativa, houve redução da motilidade. Essa aparente influência inibitória sobre o trato gastrintestinal corrobora os estudos que mostraram prejuízo no trânsito gastrintestinal de animais tratados por morfina (ROGER et al., 1994) e trabalhos com com ratos evidenciam que os receptores mu estão envolvidos central e perifericamente com analgesia, ação antissecretória e redução da motilidade (SMITH \& CHANG, 1993). DUCHARME (2003) infere que a morfina pode diminuir a motilidade progressiva do intestino delgado e do cólon, porém aumenta os movimentos de mescla e o tônus dos esfíncteres, ou seja, diminui o peristaltismo enquanto favorece o movimento segmentar e pendular, o que torna a morfina contraindicada depois de cirurgias gastrintestinais.

Cabe salientar que este estudo foi desenvolvido sob condições naturais, a fim de simular os possíveis efeitos promovidos pela morfina aplicada clinicamente em equinos. Assim, não se submeteram os animais utilizados a jejum hídrico ou alimentar previamente à administração dos fármacos. É provável que o jejum interfira na porcentagem de animais que urinaram e/ ou defecaram, na latência para urinar e/ou defecar e na motilidade intestinal.

Além disso, cabe relatar que as razões da baixa prescrição de opioides incluem seu uso ainda recente na medicina veterinária, visto que experiências mais antigas não eram animadoras, principalmente em virtude: do uso de doses diferentes das atualmente empregadas; do desconhecimento dos efeitos dos opioides diante das diferentes origens e intensidades de dor; da dificuldade de se avaliar corretamente a dor sentida pelo paciente; das deficiências no ensino médico a respeito do uso de opioides e do tratamento da dor; do medo que os médicos têm em provocar o desenvolvimento de dependência física e psíquica do paciente e as dificuldades legais para se prescrever tais fármacos (DAUDT et al., 1998; RANG et al., 2004). Entretanto, é preciso que os profissionais responsáveis pela saúde dos pacientes entendam melhor a farmacologia dos opioides, a fim de ampliarem o emprego adequado dessa classe farmacológica e usufruírem de suas vantagens terapêuticas.

\section{CONCLUSÕES}

Diante do exposto, considera-se seguro o uso intravenoso de $0,1 \mathrm{mg} / \mathrm{Kg}$ morfina em equinos da raça Puro Sangue Árabe hígidos e sem estimulação dolorosa, mas julga-se necessário que outros estudos avaliem os efeitos do uso sistêmico deste opioide no trato digestório de equinos portadores de processos dolorosos. 


\section{AGRADECIMENTOS}

À Fundação de Amparo à Pesquisa do Estado de São Paulo, (FAPESP), pelo auxílio financeiro concedido (processo ${ }^{\circ} 05 / 04480-0$ ).

\section{REFERÊNCIAS}

BRYANT, C. E.; ENGLAND, G. C.; CLARKE, K. W. Comparison of the sedative effects of medetomidine and xylazine in horses. Veterinary Records, v. 129, p. 421-423, 1991.

CAMARGO, F. C.; LEHNER, A. F.; KARPIESIUK, W.; STIRLING, K.; KAVANAGH, P. V.; BRENNAN, N.; DOWLING, M.; TOBIN, T. Review of environmental morphine identifications: worldwide occurrences and responses of authorities In: AMERICAN ASSOCIATION OF EQUINE PRACTITIONERS, 2005. Disponível em $<\mathrm{http}$ //www.ivis.org $>$. Acesso em: 11 jan. 2008.

DANNEMAN, P. J. Monitoring of analgesia. In: KOHN, D. F.; WIXSON, S. K.; WHITE, W. J.; BENSON, G. J. Anesthesia and analgesia in laboratory animals. London: Academic Press, 1997. p. 83-103.

DAUDT, A. W.; HADLICH, E.; FACIN, M. A.; APRATO, R. M. S.; PEREIRA, R. P. Opioides no manejo da dor: uso correto ou subestimado? Dados de um hospital universitário. Revista da Associação Médica Brasileira, v. 44, n. 2, p. 106-110, 1998.

DICKENSON, A. H.; SULLIVAN, A. F. Evidence for a role of the NMDA receptor in the frequency dependent potentiation of deep rat dorsal horn nociceptive neurones following $\mathrm{C}$ fibre stimulation. Neuropharmacology, v. 26, p. 1235-1238, 1987.

GAVERIAUX-RUFF, C.; KIEFFER, B. Opioid receptors: gene structure and function. In: STEIN, C. Opioids in pain control: basic and clinical aspects. Cambridge: Cambridge University Press, 1999. p. 1-21.

HELLYER, P. W. Various studies for understanding and treating acute pain in dogs and horses. In: MAYDAY CONFERENCE: A CROSS-SPECIES APPROACH TO PAIN AND ANALGESIA, 2002, Warrenton. Anais... Warrenton: Mayday Conference, 2002. Disponível em: <http://www.ivis.org>. Acesso em: 11 jan. 2008.

HUBBELL, J. A. E. Chemical restraint for standing procedures in the horse. In: NORTH AMERICAN VETERINARY CONFERENCE, 5., 2006, Orlando. Anais... Orlando: North American Veterinary Conference, 2006. Disponível em: <http:// www.ivis.org>. Acesso em: 11 jan. 2008.
KALPRAVIDH, M.; LUMB, W. V.; WRIGHT, R. B. Effects of butorphanol, flunixin, levorphanol, morphine, and xylazine in ponies. American Journal of Veterinary Research, v. 45, n. 2 , p. 217-223, 1984.

MENEZES, M. S.; GOZZANI, J. L. Analgesia pós-operatória em pacientes pediátricos: estudo comparativo entre anestésico local, opioides e antiinflamatório não esteróide. Revista Brasileira de Anestesiologia, v. 52, n. 2, p. 175-184, 2002.

MUIR III, W. W. Standing chemical restraint in horses. In: MUIR III, W. W.; HUBBELL, J. A. E. Equine anesthesia. St. Louis: Mosby Year Book, 1991. p. 247-280.

MUIR III, W. W.; HUBBELL, J. A. E.; SKARDA, R. T.; BEDNARSKI, R. M. Fármacos usados na medicação pré-anestésica. In: Manual de anestesia veterinária. 3. ed. Porto Alegre: Artmed, 2001. p. 31-44.

PASCOE, P. J.; TAYLOR, P. M. Effects of dopamine antagonists on alfentanil-induced locomotor activity in horses. Veterinary Anaesthesia and Analgesia, v. 30, p. 165-171, 2003.

RANG, H. P.; DALE, M. M.; RITTER, J. M.; MOORE, P. K. Fármacos analgésicos. In.: __ Farmacologia. 5. ed. Rio de Janeiro: Elsevier, 2004. p. $\overline{640-665 .}$

SAMMARCO, J. L.; CONZEMIUS, M. G.; PERKOWSKI, S. Z. Postoperative analgesia fot stifle surgery: a comparision of intraarticular bupivacaine, morphine or saline. Veterinary Surgery, v. 25, p. 59-69, 1996.

SCHULTZ, J. E. J.; GROSS, G. J. Opioids and cardioprotection. Pharmacology and Therapeutics, v. 89, p. 123-137, 2001.

SILVA, Y. P.; GOMEZ, R. S.; MÁXIMOM T. A.; SILVA, A. C. S. Sedação e analgesia em neonatologia. Revista Brasileira de Anestesiologia, v. 57, n. 5, p. 575-587, 2007.

TAYLOR, P. M.; CLARKE, K. W. Sedation, analgesia and premedication. In: TAYLOR, P. M.; CLARKE, K. W. Handbook of equine anaesthesia. London: W. B. Saunders, 1999. p. 15-32.

TAYLOR, P.M. Pharmacological approaches to pain management in the horse. In: ANNUAL CONVENTION OF THE AMERICAN ASSOCIATION OF EQUINE PRACTITIONERS, 51., 2005, Seattle. Anais... Seatle: American Association of Equine Practitioners, 2005. Dísponível em: $<$ http://www.ivis.org $>$. Acesso em: 11 jan. 2008.

TAYLOR, P. M.; PASCOE, P. J.; MAMA, K. R. Diagnosing and treating pain in the horse: where are we today? The Veterinary Clinics of North America. Equine Practice, v. 18, p. 1-19, 2002. 
THURMON, J. C.; TRANQUILLI, W.; BENSON, G. J. Preanesthetics and anesthetic adjunts. In.: THURMON, J. C.; TRANQUILLI, W.; BENSON, G. J. Lumb \& Jones' veterinary anestesia. Philadelphia: Lea \& Febiger, 1996. p. 183-209.
TOBIN, T. Pharmacology review: narcotic analgesics and the opiate receptor in the horse. Journal of Equine Medicine and Surgery, v. 2, p. 397-399, 1978.

Protocolado em: 13 jan. 2008. Aceito em: 24 jun. 2010. 